\title{
DERIVATION OF THE EVANS WAVE EQUATION FROM THE LAGRANGIAN AND ACTION: ORIGIN OF THE PLANCK CONSTANT IN GENERAL RELATIVITY
}

\author{
Myron W. Evans \\ Alpha Foundation for Advanced Study \\ E-mail: emyrone@aol.com
}

Received 18 November 2003

The Evans wave equation is derived from the appropriate Lagrangian and action, identifying the origin of the Planck constant $\hbar$ in general relativity. The classical Fermat principle of least time, and the classical Hamilton principle of least action, are expressed in terms of a tetrad multiplied by a phase factor $\exp (i S / \hbar)$, where $S$ is the action in general relativity. Wave (or quantum) mechanics emerges from these classical principles of general relativity for all matter and radiation fields, giving a unified theory of quantum mechanics based on differential geometry and general relativity. The phase factor $\exp (i S / \hbar)$ is an eigenfunction of the Evans wave equation and is the origin in general relativity and geometry of topological phase effects in physics, including the Aharonov-Bohm class of effects, the Berry phase, the Sagnac effect, related interferometric effects, and all physical optical effects through the Evans spin field $\mathbf{B}^{(3)}$ and the Stokes theorem in differential geometry. The Planck constant $\hbar$ is thus identified as the least amount possible of action or angular momentum or spin in the universe. This is also the origin of the fundamental Evans spin field $\mathbf{B}^{(3)}$, which is always observed in any physical optical effect. It originates in torsion, spin and the second (or spin) Casimir invariant of the Einstein group. Mass originates in the first Casimir invariant of the Einstein group. These two invariants define any particle.

Key words: Evans wave equation, Lagrangian, action, Fermat principle, Hamilton principle, topological phase effects, Evans spin field, unified theory, origin of the Planck constant and $\mathbf{B}^{(3)}$ in general relativity. 


\section{INTRODUCTION}

The Evans wave equation of unified theory [1-3] is derived from the appropriate Lagrangian density and action in general relativity. The action $S$ so defined is shown to be the origin both of the Planck constant $\hbar$ and of the Evans spin field $\mathbf{B}^{(3)}$ [4-8]. The latter is always observed in any physical optical or topological phase effect involving electromagnetism. The latter, in turn, is part of the Evans unified field theory [1-3] in general relativity and differential geometry $[9,10]$, and electromagnetism is the gauge invariant component of unified field theory originating in the torsion form of differential geometry. Gravitation is the gauge invariant component of the unified field theory originating in the Riemann form. The potential fields for both gravitation and electromagnetism originate in the tetrad $q_{\mu}^{a}$ [1-3], which is most generally defined as the invertible matrix linking two frames of reference. Section 2 identifies the Lagrangian density and action which lead to the Evans wave equation through the Euler Lagrange equation. In Sec. 3 the action defined in Sec. 2 is used to construct the phase factor

$$
\Phi=\exp (i S / \hbar)
$$

which is the origin of all topological phase effects in physics. By defining the Evans spin field $\mathbf{B}^{(3)}$ in terms of the action $S$ it is shown that the phase factor $\Phi$ is also the origin of all physical optical effects [4-8]. Inter alia, the latter are defined by $\mathbf{B}^{(3)}$ and serve to show the presence of $\mathbf{B}^{(3)}$ in any physical optical effect, the reason being that $\mathbf{B}^{(3)}$ always defines the electromagnetic phase through $S$ and the Stokes theorem of differential geometry applied to general relativity. An elegant and powerful understanding of natural philosophy is therefore possible through the use of differential geometry applied to unified field theory [1-3], and through the development of electromagnetism as a non-Abelian gauge field theory [4-8]. Section 4 unifies the Fermat principle of least time in optics and the Hamilton principle of least action in dynamics by showing that these principles of classical physics can both be defined as the tetrad multiplied by the phase factor $\Phi$. The propagation of a wave in optics or a particle in dynamics is governed essentially by $\Phi$, and the wave and particle become conceptually unified in general relativity, thus leading to the de Broglie principle of duality in general relativity and unified theory, and thus to quantum mechanics in general relativity. 


\section{THE LAGRANGIAN DENSITY AND ACTION OF THE EVANS WAVE EQUATION}

The action $S$ is the integral [9-14] with respect to the four-volume $d^{4} x$ over the Lagrangian density:

$$
S=(1 / c) \int \mathcal{L} d^{4} x,
$$

where $c$ is the speed of light in vacuo, a universal constant of general relativity. The four volume $d^{4} x$ is used because the integration takes place in a non-Euclidean spacetime of four-dimensions. The Lagrangian density is defined to be a function of the tetrad $q_{\mu}^{a}$, the derivative $\partial_{\nu} q_{\mu}^{a}$, and of $x_{\mu}$ :

$$
\mathcal{L}=\mathcal{L}\left(q_{\mu}^{a}, \partial_{\nu} q_{\mu}^{a}, x_{\mu}\right) .
$$

It follows [9-14] from Hamilton's principle of least action and the variational principle that the Lagrangian density is governed by the Euler Lagrange equation of motion:

$$
\frac{\partial \mathcal{L}}{\partial q_{a}^{\nu}}=\partial^{\mu}\left(\frac{\partial \mathcal{L}}{\partial\left(\partial^{\mu} q_{a}^{\nu}\right)}\right) .
$$

The Evans wave equation of motion [1-3] is

$$
(\square+k T) q_{\mu}^{a}=0,
$$

where

$$
T=-R / k
$$

is the index contracted energy-momentum tensor defined through Eq. (6) by the negative of the scalar curvature $R$ divided by $k$, the Einstein constant. In Eq. (5) $\square$ is the d'Alembertian operator with respect to Euclidean spacetime [1-3]:

$$
\square=\frac{1}{c^{2}} \frac{\partial^{2}}{\partial t^{2}}-\frac{\partial^{2}}{\partial X^{2}}-\frac{\partial^{2}}{\partial Y^{2}}-\frac{\partial^{2}}{\partial Z^{2}} .
$$

Equation (5) follows from the Eq. (4) given the Lagrangian density

$$
\mathcal{L}=-\frac{c^{2}}{k}\left[\frac{1}{2}\left(\partial_{\mu} q_{\nu}^{a}\right)\left(\partial^{\mu} q_{a}^{\nu}\right)+\frac{R}{2} q_{\nu}^{a} q_{a}^{\nu}\right]=-\frac{R}{k} c^{2} .
$$

Using Eq. (8), the left-hand side of Eq. (4) becomes

$$
\partial \mathcal{L} / \partial q_{a}^{\nu}=-\frac{R c^{2}}{k} q_{\nu}^{a},
$$


and the right-hand side is

$$
\partial \mathcal{L} /\left(\partial\left(\partial^{\mu} q_{a}^{\nu}\right)\right)=-\frac{c^{2}}{k} \partial_{\mu} q_{\nu}^{a} .
$$

The Euler-Lagrange equations (4) therefore gives the Evans lemma [15] or subsidiary proposition of differential geometry:

$$
\square q_{\nu}^{a}=R q_{\nu}^{a},
$$

given the Lagrangian density (8). The Evans wave equation (5) follows from the lemma (11) using Eq. (6). The Lagrangian density (8) also defines the scalar curvature in terms of the derivative of the tetrad:

$$
R=\partial_{\mu} q_{\nu}^{a} \partial^{\mu} q_{a}^{\nu}
$$

The Evans wave equation and lemma are derived [1-3] from the tetrad postulate of differential geometry $[9,10]$ :

$$
D_{\mu} q_{\lambda}^{a}=\partial_{\mu} q_{\lambda}^{a}+\omega_{\mu b}^{a} q_{\lambda}^{b}-\Gamma_{\mu \lambda}^{\nu} q_{\nu}^{a}=0 .
$$

Using Eq. (13) in Eq. (12) defines the scalar curvature $R$ (and thus $T$ ) in terms of the Christoffel connection $\Gamma_{\mu \lambda}^{\nu}$ and the spin connection $\omega_{\mu b}^{a}$ for any spacetime:

$$
R=\left(\Gamma_{\mu \lambda}^{\nu} q_{\nu}^{a}-\omega_{\mu b}^{a} q_{\lambda}^{b}\right)\left(\Gamma_{\nu}^{\mu \lambda} q_{\nu}^{a}-\omega_{a}^{\mu b} q_{b}^{\lambda}\right)
$$

This definition of the scalar curvature follows from the assumption that the Lagrangian density is a function of three variables of the type (3). It shows that for any non-zero $T$ the connections must also be non-zero, meaning that the spacetime is never Euclidean, and that the spacetime always contains both curvature and torsion. This is a major advance in understanding over the original theory of general relativity [15], which applies only to gravitation, and in which the torsion tensor is set to zero. For this reason the original theory of general relativity [15] cannot define electromagnetism.

In Eq. (5) $T$ is the total energy in general. When there is no potential energy, the total energy and the Lagrangian are identical and given by the kinetic energy:

$$
T=L=E_{\mathrm{kin}} .
$$

More generally, the Hamiltonian and Lagrangian are defined by

$$
\begin{aligned}
& H=E_{\text {kin }}+E_{\text {pot }}, \\
& L=E_{\text {kin }}-E_{\text {pot }} .
\end{aligned}
$$


When considering the Evans lemma and wave equation, the effect of potential energy or interaction energy is always to change $R$. This means that Feynman diagrams, for example, could be replaced by a theory depending on changes of $R$, a theory which contains no singularities. This would be a major advance in areas such as quantum electrodynamics. In general the Evans equation would be solved numerically for any given problem in physics and general relativity using powerful contemporary code and code libraries for the solution of partial, second-order, differential equations (wave equation).

\section{PHASE FACTOR, TOPOLOGICAL EFFECTS, AND ORIGIN OF THE PLANCK CONSTANT IN GENERAL RELATIVITY}

For a free particle (or field) there is only kinetic energy present and so total energy $T$ and Lagrangian density originate in kinetic energy. This means that the Lagrangian density is defined in terms of $R(=-k T)$ using Eq. (8). The action for a free particle is therefore

$$
S=-\frac{c}{k} \int R d^{4} x
$$

The action is an integral over the scalar curvature. This result remains true in the presence of potential or interaction energy provided the scalar curvature $R$ is changed, and provided the Lagrangian is defined correctly by Eq. (17). This result may be summarized in the following theorem:

If the scalar curvature is defined by

$$
T=-R / k
$$

then

$$
\int R d^{4} x=-\frac{k}{c} S
$$

The phase factor of the Evans unified field/matter theory can therefore always be defined by a scalar curvature for any situation in physics:

$$
\Phi=\exp (i S / \hbar)=\exp \left(\frac{-i c}{\hbar k} \int R d^{4} x\right) .
$$

As shown in Sec. 4, the classical Fermat and Hamilton principles are defined in turn by Eq. (21), thus providing a powerful basis for the development of quantum mechanics from general relativity and differential geometry. The origin of these principles of classical physics is 
found from the fact that the eigenfunction of the Evans wave equation can always be written as the tetrad

$$
q_{\mu}^{a}(x)=\Phi q_{\mu}^{a}(0)
$$

Application of the Leibnitz theorem [16] to Eq. (22) shows that

$$
(\square+k T) \Phi=0,
$$

and so the phase factor $\Phi$ itself gives curvature and energy eigenvalues from the wave equation (26). This is the origin of the well observed topological phase effects $[17,18]$ in physics. Examples are the Aharonov Bohm class of effects, the Berry phase in quantum mechanics, the Sagnac effect in physical optics, the closely related Tomita Ciao effect [4-8] and in general all physical optical effects, because the latter all depend on light propagation, and so they all depend on the phase defined in Eq. (21) through the Fermat principle of least time [19]. A gauge transformation can also be understood as a phase effect dependent on $\Phi$, and so $\Phi$ is related to the rotation operator in the gauge transformation of the general $n$-dimensional field [22]. Therefore all these effects become understandable as effects of general relativity, as required by the principle of relativity of Einstein, and not of special relativity. The principle of relativity requires that all theories of physics be theories of general relativity, and ultimately, all topological phase effects can be traced, through the Evans wave equation, to the non-trivial topology of spacetime itself. This topology is summarized in $R(=-k T)$, and the phase (21) is defined by an integral over $R$.

The global or gauge-invariant phase factor is defined through the Dirac or Wu-Yang phase factor $[17,18]$ :

$$
\Phi^{a}=\exp \left(i e \oint A_{\mu}^{a} d x^{\mu}\right) .
$$

The physical quantity in Wu-Yang-Dirac phase is $e$ multiplied by the gauge invariant contour integral over the electromagnetic potential $A_{\mu}^{a}$. The latter is related through the Stokes theorem to the surface integral over the gauge invariant electromagnetic field. The gauge invariant and non-oscillatory (i.e., phase free) electromagnetic field component in the Wu-Yang-Dirac phase is the Evans spin field $\mathbf{B}^{(3)}$ [4-8]. This means that the action can be defined through the Stokes theorem and in general relativity and unified field theory the action is defined in this way for any radiated or matter field.

The conventional [14] definition of a matter or radiated wave is

$$
\psi=\psi_{0} \exp \left(i \kappa_{\mu} x^{\mu}\right)
$$


but this definition is incorrect, because it is invariant under parity inversion

$$
\hat{P}\left(x_{\mu} \kappa^{\mu}\right)=x_{\mu} \kappa^{\mu},
$$

and for this reason cannot provide even a qualitative description of ordinary effects in physical optics such as reflection (i.e., parity inversion) and interferometry [8]. If the phase is invariant under reflection there cannot be an observed reflection. This simple fact appears to have been overlooked in conventional physical optics. In order to construct a Michelson interferogram, for example [4-8], the phase must change sign under $\hat{P}$ at the point of beam reflection from the mirrors of the Michelson interferometer, and this property is given by the contour integral definition of phase used in the Wu-Yang-Dirac phase factor of electrodynamics:

$$
\psi=\psi_{0} \exp \left(i \oint \kappa_{\mu} d x^{\mu}\right)
$$

This is an important development in physical optics because the Stokes theorem always relates the contour integral to a surface integral, showing that spin is always present in the phase of a matter wave (such as an electron beam) or a radiated wave (such as an electromagnetic beam). In the conventional description of the phase only the energy-momentum is present, represented by $\hbar \kappa^{\mu}$. The conventional description is incomplete essentially because there are two invariants of the Einstein group, mass and spin, and both are needed to define a particle such as an electron or photon. The fundamental spin field in the electromagnetic phase in general relativity is the $\mathbf{B}^{(3)}$ field, which appears in the surface or area integral of the Stokes theorem. In the notation of differential geometry $[9,10]$ the latter means that

$$
\oint \kappa_{\mu} d x^{\mu}=\int(d \wedge \kappa)_{\mu \nu} d \sigma^{\mu \nu}
$$

or, in condensed notation,

$$
\oint_{\delta S} \kappa=\int_{S} d \wedge \kappa
$$

where $d^{\wedge}$ is the exterior derivative. Eq. (29) is true for all geometries of the base manifold, and so is true in general relativity, which is the geometrical theory of all physics.

If $q^{a}$ is the tetrad form (a vector valued one form), then the Stokes theorem implies that

$$
\oint_{\delta S} q^{a}=\int_{S} \tau^{a}
$$


where

$$
\tau^{a}=d \wedge q^{a}
$$

is the torsion form [1-3,9,10] as defined by the first Maurer-Cartan structure relation of differential geometry $[9,10]$. So the Stokes theorem relates the contour integral over the tetrad form to the surface integral over the torsion form for any geometry of the base manifold:

$$
\oint q_{\mu}^{a} d x^{\mu}=\int \tau_{\mu \nu}^{a} d v^{\mu \nu}
$$

The torsion form in unified field theory [1-3] is the electromagnetic field within a factor $A^{(0)}$ with the units of weber $\mathrm{m}^{-1}=$ volts $\mathrm{s}^{-1} \mathrm{~m}^{-1}$ and the tetrad form is the electromagnetic potential field [1-3]:

$$
A_{\mu}^{a}=A^{(0)} q_{\mu}^{a}
$$

where $A^{(0)}$ is a potential magnitude. Therefore in unified field theory the Stokes theorem (30) inter-relates the potential and gauge-invariant fields:

$$
\oint_{\delta S} A^{a}=\int_{S} B^{a}=\int_{S} d \wedge A^{a} .
$$

The potential field itself is not gauge invariant, but the contour integral over the potential field is gauge invariant. In the Aharonov-Bohm effect [14], gauge transformation of the potential field produces a physical effect, so both the potential and the gauge invariant fields are physically meaningful in classical general relativity as well as quantum mechanics. The unified field theory [1-3] has shown that quantum mechanics emerges from differential geometry, (see Sec. 4), so the gauge transformation of the second kind produces effects such as entanglement, which are conventionally explained in terms of quantum mechanical wavefunctions without classical meaning. Unified field theory [1-3] therefore traces the origin of entanglement [8] to the gauge transformation of the second kind. Action at a distance is therefore not needed to explain entanglement.

The Wu-Yang-Dirac phase developed for general relativity then follows from Eq. (34):

$$
\exp \left(i \frac{e}{\hbar} \oint A^{a}\right)=\exp \left(i \frac{e}{\hbar} \int B^{a}\right)
$$

and completely defines the electromagnetic field because this phase is gauge invariant for all geometries of the base manifold. In contrast, the conventional phase factor of Maxwell-Heaviside theory is not gaugeinvariant:

$$
\Phi_{\mathrm{MH}}=\exp [i(\omega t-\boldsymbol{\kappa} \cdot \mathbf{r}+\alpha)],
$$


so an arbitrary factor $\alpha$ can be added [17] to it without affecting physical optics. This description of nature is therefore over determined. It also fails to give a qualitative account of reflection and interferometry as argued already.

This development suggests that the phase for radiated and matter waves is defined in general relativity by the tetrad form of differential geometry:

$$
\exp \left(i \kappa \oint_{\delta S} q^{a}\right)=\exp \left(i \kappa \int_{S} d \wedge q^{a}\right)
$$

for all possible geometries of the base manifold. The index $a$ defines the tangent space basis, which is orthogonal and normalized, i.e., an Euclidean space. If we make the gauge transformation of the second kind $[8]$ :

$$
q^{a} \rightarrow q^{a}+\frac{1}{\kappa} d \chi^{a}
$$

where $d$ denotes the ordinary (not exterior) derivative, then

$$
d \wedge q^{a} \rightarrow d \wedge q^{a}+\frac{1}{\kappa} d \wedge d \chi^{a}=d \wedge q^{a}
$$

on using the Poincaré lemma

$$
d \wedge d:=0
$$

This result shows that the right-hand side of Eq. (37) is gauge invariant. Therefore the left-hand side must also be gauge invariant, implying that

$$
\oint d \chi^{a}:=0
$$

for all geometries of the base manifold. Eq. (41) is true for all $\chi^{a}$, irrespective of whether $\chi^{a}$ is single valued or periodic (integral or multivalued). Therefore the Aharonov-Bohm effect, for example, cannot be described by the Stokes theorem (34), and the Aharonov-Bohm effect cannot be understood with the conventional phase (36), showing in another way that that phase is unphysical. Recall that the phase (36) cannot be used to understand reflection or interferometry in physical optics, and cannot be used to understand interferometry in matter waves, such as the Sagnac effect in electron beams [8]. The conventional description of the Aharonov-Bohm effect [14] contradicts the fundamental result (41) from gauge invariance, and the conventional description is therefore incorrect. 
This fundamental paradox of conventional electromagnetic theory is resolved, however, by developing the Stokes theorem with the covariant exterior derivative $[9,10,18]$ denoted by $D^{\wedge}$ :

$$
\oint_{\Delta S} q^{a}=\int_{S} D \wedge q^{a}
$$

where

$$
D \wedge q^{a}=d \wedge q^{a}+\kappa q^{b} \wedge q^{c} .
$$

Equation (43) is the non-Abelian Stokes theorem $[8,17]$ in the notation of differential geometry. The covariant exterior derivative acts on a differential $n$ form to produce a differential $n+1$ form which transforms properly under general coordinate transformation $[9,10]$, i.e., as a proper tensor. The ordinary exterior derivatives produces a vector valued $n+1$ form from a vector valued $n$ form. The $n+1$ form transforms correctly under the transformation law for $(0,2)$ tensors for general coordinate transformations, but does not transform correctly as a vector under linear Lorentz transformations $[9,10]$. This flaw in the $d^{\wedge}$ operator is remedied by replacing it by the $D^{\wedge}$ operator, the covariant exterior derivative operator of differential geometry. The latter acts on the vector valued one form $X_{\mu}^{a}$, for example the tetrad, to produce $[9,10]$ the vector-valued two-form

$$
\begin{aligned}
(D \wedge X)_{\mu \nu}^{a} & =(d \wedge X)_{\mu \nu}^{a}+(\omega \wedge X)_{\mu \nu}^{a} \\
& =\partial_{\mu} X_{\nu}^{a}-\partial_{\nu} X_{\mu}^{a}+\omega_{\mu b}^{a} X_{\nu}^{b}-\omega_{\nu b}^{a} X_{\mu}^{b}
\end{aligned}
$$

where $\omega_{\mu b}^{a}$ is the spin connection. Recent work [3] has shown that the spin connection is the Hodge dual of the tetrad, and that the gauge invariant Riemann form of gravitation is the Hodge dual of the gauge invariant torsion form of electromagnetism [1-3]. The object $D^{\wedge} X^{a}$ always transforms as a proper tensor $[9,10]$ for all spacetime geometries of the base manifold, and therefore for all situations in general relativity and unified field theory. The unified field theory [1-3] traces the origin of both gravitation and electromagnetism to differential geometry, thus implying the use of $D^{\wedge}$ for basic mathematical correctness. The replacement of $d^{\wedge}$ by $D^{\wedge}$ produces the $\mathbf{B}^{(3)}$ field [1-8,17], produces the correct explanation of reflection and interferometry through the phase constructed from (42), and clearly explains the Aharonov-Bohm effect as follows.

The electromagnetic potential field in the unified field theory is defined as the tetrad [1-3]

$$
A_{\mu}^{a}=A^{(0)} q_{\mu}^{a}
$$


where $A^{(0)}$ is the scalar magnitude of the fundamental, $C$ negative, potential field, whose origin can be traced to primordial magnetic flux in units of volts $\mathrm{s}^{-1}[1-3]$. So, in generally covariant electrodynamics $[4-8]$,

$$
D \wedge A^{a}=d \wedge A^{a}+g A^{b} \wedge A^{c},
$$

with

$$
g=e / \hbar=\kappa / A^{(0)}
$$

as the fundamental charge $e$ on the proton divided by the Planck constant $\hbar$. Thus $g$ is a universal constant [4-8] that defines the photon momentum by

$$
p=\hbar \kappa=e A^{(0)},
$$

and the origin of $g$ can be traced to differential geometry, the need for a $D^{\wedge}$ operator instead of a $d^{\wedge}$ operator as argued already. Thus, general relativity and unified field theory show the need for $g$ in electrodynamics. In conventional Maxwell-Heaviside theory (a theory of special relativity) $g$ is missing, essentially because spacetime in the Maxwell Heaviside theory is flat or Euclidean and the spin connection and $R$ are both zero. In general relativity this implies that the universe is devoid of all matter fields, all radiation fields and all energy-momentum ( $T$ is zero for all $k$ if $R$ is zero). General relativity and differential geometry imply the $\mathbf{B}^{(3)}$ field, defined as the differential form

$$
B^{(3)}=-i g A^{(1)} \wedge A^{(2)} .
$$

(If $g$ were zero (flat spacetime) $\mathbf{B}^{(3)}$ would be zero, but as just argued, $\mathbf{B}^{(3)}$ is responsible for all physical optics.) The correctly covariant definition of the $\mathrm{Wu}$-Yang phase in general relativity is therefore

$$
\Phi=\exp \left(i g \oint_{D S} A^{a}\right)=\exp \left(i g \int_{S} B^{a}\right)=\exp \left(i g \int_{S} D \wedge A^{a}\right) .
$$

In order to understand the Aharonov-Bohm class of effects, consider the property of the covariant Stokes theorem

$$
\oint_{D S} A=\int_{S} D \wedge A
$$

under the local gauge transformation

$$
A^{\prime}=\sigma A,
$$

where $\sigma$ is rotation operator [9]. In Eqs. (51) and (52) we have used the generic notation of differential geometry. Covariant differentiation of the gauge transform relation (52) produces

$$
D^{\prime} A^{\prime}=\sigma D A
$$


where

$$
\begin{gathered}
D=d+g A, \\
D^{\prime}=d+g A^{\prime},
\end{gathered}
$$

while ordinary differentiation leads to

$$
d A^{\prime}=d(\sigma A)=\sigma d A+A d \sigma .
$$

From Eqs. (54) and (55) inserted in (53),

$$
d A^{\prime}+g A^{\prime} A^{\prime}=\sigma d A+g \sigma A A .
$$

Using Eq. (56) in (57),

$$
\begin{aligned}
\sigma d A+A d \sigma+g A^{\prime} A^{\prime} & =\sigma d A+g \sigma A A \\
A d \sigma+g A^{\prime} A^{\prime} & =g \sigma A A
\end{aligned}
$$

and, using Eq. (52) in (58),

$$
\begin{aligned}
& A d \sigma+g A^{\prime} \sigma A=g \sigma A A, \\
& A^{\prime}=\sigma A \sigma^{-1}-\frac{1}{g} d \sigma \sigma^{-1} .
\end{aligned}
$$

This equation defines the change in the tetrad form $A_{\mu}^{a}$ (the electromagnetic potential form) under the local gauge transformation (52), also known as the gauge transform of the second kind $[8,9]$. This is the relativistically correct gauge transform and is the basis for all gauge field theory in physics because it leaves the action invariant, and leads to fundamental conservation theorems such as the Noether theorem.

In conventional electromagnetic theory [9] the electromagnetic field is defined by the ordinary exterior derivative acting on the scalarvalued one-form $A$ :

$$
F=d \wedge A,
$$

and the field is invariant under the gauge transform (6):

$$
F^{\prime}=d \wedge A^{\prime}=d \wedge A-(1 / g \sigma) d \wedge d \sigma=d \wedge A .
$$

It follows from the Stokes theorem of conventional electromagnetic theory,

$$
\oint_{\delta S} A=\int_{S} d \wedge A,
$$


that there is no Aharonov-Bohm effect in conventional electromagnetic theory. The reason is that the right-hand side of Eq. (63) is unchanged (i.e., invariant) under the gauge transform (60), so

$$
\oint_{\delta S} d \sigma=\int_{S} d \wedge d \sigma:=0
$$

for all $\sigma$, irrespective of whether $\sigma$ is a single-valued or periodic (multivalued) function. The conventional explanation of the Aharonov-Bohm effect [9] incorrectly [8] asserts however that

$$
\oint_{\delta S} d \sigma \neq 0(?)
$$

The correctly and generally covariant electromagnetic field [1-3] transforms as

$$
G^{\prime}=D^{\prime} \wedge A^{\prime}=d \wedge A^{\prime}+g A^{\prime} \wedge A^{\prime}
$$

under the frame rotation induced by $\sigma$ as described in Eq. (52). Using the result

$$
d \wedge A=d \wedge A^{\prime}
$$

it is seen that the rotation produces the effect

$$
A \wedge A \rightarrow A^{\prime} \wedge A^{\prime}
$$

which means that the magnetic field defined in Eq. (49) transforms under local gauge transformation, i.e., the rotation (52), as

$$
B=-i g A \wedge A \rightarrow-i g A^{\prime} \wedge A^{\prime} .
$$

This transformation is the origin of the Aharonov-Bohm effect, which is therefore due to general relativity, not special relativity as in the (incorrect) conventional theory.

The definition (69) of the magnetic field in terms of the cross product of two potentials was first inferred [4-8] for the radiated EvansVigier field $\mathbf{B}^{(3)}$ (now known to be the fundamental spin Casimir invariant of the Einstein group in electrodynamics), but the definition has been extended [4-8] to a static (non-radiated) magnetic field because the correctly and generally covariant definition of the electric and magnetic fields must always be

$$
G=D \wedge A
$$

in which $d^{\wedge}$ has been replaced by $D^{\wedge}$. As argued already, this replacement is a fundamental requisite of differential geometry. The exterior derivative of the potential $(d \wedge A)$ as in conventional electromagnetic 
theory (special relativity) is replaced by the covariant exterior derivative of the tetrad $(D \wedge A)$ in general relativity. In the Aharonov-Bohm effect, regions of the experimental set up are considered where the original magnetic flux density and original potential are both zero. In these regions an electromagnetic effect is nevertheless observed, for example as a shift in an electron diffraction pattern. The Aharonov-Bohm effect originates in the magnetic flux

$$
B^{\prime}=-i g\left(\frac{1}{g^{2} \sigma^{2}} d \sigma \wedge d \sigma\right)
$$

which is purely geometrical or topological in nature and which does not depend on the original potential $A$. The magnetic flux that is observed in the effect is

$$
\phi=\int B^{\prime} d A r
$$

and so the original magnetic flux density has been shifted by the local gauge transform (52) into other regions of spacetime. This effect is due to the structure of spacetime itself, and therefore due to general relativity. It is obviously not due to action at a distance, a concept which violates relativity and therefore violates the Noether theorem, which as argued already is derived from relativity through local gauge transformation. Anything that violates the Noether theorem violates conservation of energy, momentum, charge and current. The gauge transform (52) is defined by the complex-valued rotation operator

$$
\sigma=\exp \left(i \Lambda^{a}\left(x^{\mu}\right) M^{a}\right)
$$

and so

$$
\left|A^{\prime} \wedge A^{\prime}\right|=|A \wedge A|,
$$

by the property of complex conjugation.

It is worth emphasizing that the basic error in the conventional theory of the Aharonov-Bohm effect [9] can be understood clearly in the notation of differential geometry as follows:

$$
\begin{gathered}
A \rightarrow A+\frac{1}{g} d \chi \\
\oint_{\delta S} d \chi=\int_{S} d \wedge d \chi:=0 .
\end{gathered}
$$

However, it is incorrectly asserted in the original theory [9] that

$$
\oint_{\delta S} d \chi \neq 0(?)
$$


This mathematical error was first pointed out in Ref. [8] and is now easily understood using differential geometry as argued already.

A generally covariant theory of electrodynamics is therefore needed to correctly explain the Aharonov-Bohm effect in terms of $D \wedge A$. In the correctly covariant Stokes theorem (51), gauge transformation (52) produces the result

$$
\oint_{D S} d \sigma=\frac{i}{\sigma} \int_{S} d \sigma \wedge d \sigma \neq 0
$$

in regions where the original magnetic flux density and potential are both zero. There is therefore an electromagnetic effect in these regions as observed. The unified field theory [1-3] means that there must be a gravitational analogy of the Aharonov-Bohm effect, and such an effect is indeed observed - the well-known Coriolis and centripetal acceleration due to a rotating frame of reference. The rotation of the frame is a local, or relativistically correct, gauge transformation of type two observed in the weak field limit. These accelerations are observed where there is no Newtonian (or central or linearly directed) acceleration, and therefore no Newtonian gravity and no Newtonian force. It is nevertheless clear that the centripetal and Coriolis accelerations are physical accelerations that exist in regions where there is no Newtonian acceleration, and which are generated by a gauge or frame transformation in the weak field limit of general relativity. They therefore meet all the requirements of an Aharonov-Bohm effect.

The fundamental geometrical reason for the Aharonov-Bohm effect is that the Stokes theorem must be generally covariant, as in Eq. (51). Such a Stokes theorem is mathematically non-Abelian [8], and has properties in general relativity and unified field theory that are not present in special relativity. One of these is the AharonovBohm effect as just argued, others include the topological phase effects, the Sagnac effect, and indeed all physical optics, because the phase in electrodynamics can only be explained correctly with the non-Abelian or generally covariant Stokes theorem [8] (Eq. (50)). The latter can always be written as

$$
\oint_{D S} A^{a}=\int_{S} d \wedge A^{a}+g \int_{S} A^{b} \wedge A^{c}
$$

If $a=(1),(2),(3)$, the labels of the complex circular basis of circularly polarized electromagnetic radiation [4-8], then

$$
\oint_{D S} A^{(i)}=\int_{S} d \wedge A^{(i)}, \quad i=1,2
$$

for the transverse components (1) and (2), and

$$
\oint_{D S} A^{(3)}=-i g \int_{S} A^{(1)} \wedge A^{(2)}=\int_{S} B^{(3)}
$$


for the longitudinal component (3). In the conventional theory of electromagnetism the transverse components are radiated plane waves and the longitudinal component is missing. The fundamental reason for this is that the conventional theory is an incorrectly Abelian gauge field theory in which the phase is incorrectly defined. In the generally covariant theory of electrodynamics [1-3] however, the (3) component of gauge transformation from a region 1 to a region 2 can always be written (From Eq. (80) [4-8]) as

$$
A_{1}^{(3)} \rightarrow A_{2}^{(3)^{\prime}} .
$$

Under this gauge transformation the magnetic flux density is invariant, but is shifted from region 1 to region 2 , so the effect can be traced to a property of spacetime itself in general relativity. In special relativity, the spacetime is flat or Euclidean, and the Aharonov-Bohm effect cannot be explained with flat spacetime. Similarly the action

$$
S=e \oint_{D S} A_{1}^{(3)} \rightarrow e \oint_{D S} A_{2}^{(3)^{\prime}}
$$

is invariant under the longitudinal, local gauge transform (52) but the action is shifted from region 1 to region 2. This shift in the action is accompanied by a shift in the generally covariant electromagnetic phase factor

$$
\Phi=\exp (i S / \hbar) .
$$

The local gauge transformation responsible for this shift in the phase factor is a frame rotation defined by the rotation operator in Eq. (73). In the Sagnac effect, a corresponding shift in phase factor is brought about by a physical rotation of the platform of the Sagnac interferometer [8] and the Sagnac effect can be understood [8] as a phase shift brought about by an increase or decrease in the wave number $(\kappa=\omega / c)$ which is related to the longitudinal potential component in the phase factor (50) by

$$
\kappa^{a}=g A^{a} .
$$

Therefore the shift (82) can be understood in the Sagnac effect as a frequency shift

$$
\omega \rightarrow \omega \pm \Omega .
$$

In Eq. (85) $\Omega$ is the angular frequency of the rotating platform and the plus or minus signs originate from clockwise and anticlockwise platform rotation respectively. In the Aharonov-Bohm effect the area $A r$ in the surface integral on the right-hand side of Eq. (50) is defined by the area enclosed by diffracting beams in a Young or two-slit interferometer. In the Sagnac effect the corresponding area is defined by the area enclosed by the Sagnac interferometer, i.e., by the paths of the electromagnetic beams or matter beams such as an electron beam or molecular beam 
on the platform, either at rest or rotating at angular frequency, $\Omega$. The Sagnac effect is explained as follows from the generally covariant phase factor (50).

The magnitude of $B^{a}$ is defined by [4-8]

$$
B^{(0)}=\kappa A^{(0)} .
$$

The phase factor (50) therefore becomes

$$
\Phi=\exp \left(i \frac{e}{\hbar} \oint \mathbf{A}^{(0)} \cdot d \mathbf{r}\right)=\exp \left(i \frac{e}{\hbar} \kappa A^{(0)} \int d A r\right)
$$

and can be written as

$$
\Phi=\exp \left(i \oint \boldsymbol{\kappa}^{(3)} \cdot d \mathbf{r}\right)=\exp \left(i \kappa^{2} A r\right)
$$

The Sagnac effect with platform at rest is given by the area integral on the right-hand side, which is equal to the contour integral around the boundary of this area on the left-hand side. If the area is a circle the boundary is the circumference. The interferogram or diffraction pattern of the Sagnac effect with platform at rest is therefore

$$
\operatorname{Re}(\Phi)=\cos \left(\kappa^{2} A r\right)=\cos \left(\omega^{2} / c^{2} A r\right)
$$

as observed experimentally to one part in $10^{23}$ precision [8]. The Sagnac effect from Eq. (89) depends on the magnitude of the area enclosed by the boundary, but not to the shape of the boundary, and this is again as observed experimentally. Thirdly, the Sagnac effect with platform at rest originates in the generally invariant phase factor

$$
\operatorname{Re}(\Phi)=\cos \left(\omega^{2} / c^{2} A r\right)
$$

(a scalar frequency squared divided by $c^{2}$ and multiplied by a scalar magnitude of area), and so the Sagnac effect with platform at rest is the same for an observer on and off the platform, again as observed experimentally [8]. Similarly the contour integral on the left-hand side of Eq. (88) is the same to an observer on and off the platform. The Sagnac effect with platform in motion is given by the interferogram

$$
\operatorname{Re}\left(\Phi_{m}\right)=\cos \left(\left((\omega+\Omega)^{2}-(\omega-\Omega)^{2}\right) \frac{A r}{c^{2}}\right)=\cos \left(4 \frac{\omega \Omega}{c^{2}} A r\right),
$$

as observed experimentally to one part in $10^{23}$.

In conventional electrodynamics (Maxwell-Heaviside theory) there is no Sagnac effect [8], because that theory is invariant under 
motion reversal $T$, and is also metric invariant, i.e., a theory of flat spacetime. In generally covariant electrodynamics [1-4] the Sagnac effect is described by the generally covariant phase factor (50), which is also the origin of the Wu-Yang phase factor. The Sagnac effect can only be described if it is recognised that the torsion form in gravitational theory is the origin of the Coriolis and centripetal accelerations, and may be the origin of dark matter in the universe.

The electromagnetic phase in general relativity and unified field theory is therefore

$$
\Phi=\exp \left(i \frac{e}{\hbar} \oint A^{(3)} d Z\right)=\exp \left(i \frac{e}{\hbar} \int B^{(3)} d A r\right)
$$

and the following equations define the magnitudes $B^{(0)}$ and $A^{(0)}$, respectively, in terms of $R$ and $\kappa$ :

$$
\begin{aligned}
& e B^{(0)}=\hbar R, \\
& e A^{(0)}=\hbar \kappa, \\
& B^{(3)}=\frac{\hbar}{e} R .
\end{aligned}
$$

Similarly the mass is defined by

$$
m=-\int \frac{R}{\kappa} d V
$$

These equations show that mass $m$ is the first (mass) Casimir invariant of the Einstein group, and the $\mathbf{B}^{(3)}$ field is the second (spin) Casimir invariant of the Einstein group within the scalar magnitude $B^{(0)}$. The corresponding conclusions hold in special relativity if the Einstein group is replaced by the Poincaré group, as first demonstrated by Wigner [8].

The $\mathbf{B}^{(3)}$ or Evans-Vigier field originates therefore in the fundamental spin invariant of general relativity, mass being the other fundamental invariant.

The magnitude of the photon momentum is defined by

$$
\rho=\hbar \kappa=e A^{(0)}
$$

and so $e$ is a primordial charge (with the magnitude of the charge on the proton) present within the radiated electromagnetic field. So the photon is both a particle and a field. The $g$ of non-Abelian gauge field theory [4-8] applied to the electromagnetic field is $e / \hbar$. With 
these definitions, the electromagnetic phase factor in general relativity is therefore

$$
\begin{aligned}
\Phi & =\exp (i S / \hbar)=\exp \left(i \int \mathcal{L} d^{4} x\right) \\
& =\exp \left(-\frac{i}{k} \int R d^{4} x\right) \\
& =\exp (i \kappa \oint d Z)=\exp \left(i \kappa^{2} \int d A r\right), \\
R & =\kappa^{2} .
\end{aligned}
$$

The Planck constant is the least amount of action or angular momentum present in the universe, and so is defined in terms of scalar curvature by

$$
\hbar=-\frac{c}{r} \int R_{0} d^{4} x
$$

The local gauge transformation (52) is the generation of one tetrad, $A_{\mu}^{\prime a}$, from another, $A_{\mu}^{a}$,

$$
A_{\mu}^{\prime a}=\sigma A_{\mu}^{a}
$$

and is therefore a form of the Fermat principle of least time or Hamilton principle of least action combined into one equation (100) of unified field theory expressed by differential geometry. In Eq. (100) the action $S$ is automatically invariant under the gauge transform because the latter is defined in terms of rotation generators of a given gauge group, so we obtain an equation of general relativity defining the invariant action in terms of the rotation generator

$$
S=\hbar M^{a} \Lambda^{a}
$$

for any given gauge group and any given representation space of the base manifold and for any geometry of the base manifold.

It is well known [19] that Fermat's principle of least time governs all physical optics, so it must also govern the Aharonov-Bohm and Sagnac effects, as just shown. Using Eq. (98) the Sagnac effect for example can now be understood in general relativity and unified field theory [1-3] as a change in scalar curvature of spacetime produced by rotating the platform in the Sagnac experiment. The Sagnac effect observed [8] in electron beams can be understood in the same way; it is fundamentally a change in phase produced by the scalar curvature of spacetime, and can be observed both in matter waves (electrons or molecules for example) and radiated waves (visible frequency light for example). The Sagnac effect observed in radiated and matter fields is therefore proof of the fundamental relations (98), and shows that all 
physical optical effects of the electromagnetic field are produced by the fundamental $\mathbf{B}^{(3)}$ field [8]. In matter waves the $\mathbf{B}^{(3)}$ field becomes the fundamental spin of the particle making up the matter field (for example the electron). The Sagnac effect therefore constitutes proof of the Evans unified field theory, in which particle spin from the Dirac equation is deduced from the Evans wave equation [1-3]. The latter applies to both radiated and matter fields. The Sagnac effect in matter fields is also an experimental demonstration of quantum mechanics, and unifies the Fermat and Hamilton principles through Eq. (22), producing the de Broglie wave particle duality. In the closely related Tomita-Chao effect [8] an increment of the phase (98) or (92) is detected after an electromagnetic beam propagates through several loops of a helix using an optical fiber. In the Berry effects [14] a similar type of geometrically generated phase (98) is detected after an electromagnetic beam propagates through several loops of a helix using an optical fiber. In both experiments the phase increment is described by a contour integral, so is additive if the light propagates through several loops. The BohmAharonov effect is detected with a two-aperture interferometer and is described by the same equation (98) as the Sagnac effect. The only difference between the two effects is geometry and the way in which an observable phase shift is induced: the former by a frame rotation, the latter by a physical rotation. In a physical optical effect such as reflection or in an interferometric effect such as Michelson interferometry [8], the effect being observed is described in the same way, mathematically, as in the Sagnac and Aharonov-Bohm effects. All these effects [8] are manifestations of Eq. (98), in which the phase factor and action are defined with the Stokes theorem, and manifestation of the Fermat principle of least time, Eq. (22). The existence of Michelson interferometry (and all types of interferometry) depends [8] on the following property of the contour integral within the exponent of the phase factor:

$$
\oint_{0 A} \mathbf{A} \cdot d \mathbf{r}=-\oint_{A 0} \mathbf{A} \cdot d \mathbf{r}
$$

In an electromagnetic wave propagating along the $Z$ axis, this contour integral is valued along the following closed boundaries defining an area. If the distance $0 A$ is $n$ times the wavelength $\lambda$, then the area enclosed [8] is $n \lambda^{2} / \pi$. The change in phase on normal reflection is then [8]

$$
\exp (i \oint \kappa d Z)=\exp \left(i \int_{0}^{Z} \kappa d Z-i \int_{Z}^{0} \kappa d Z\right)=\exp (2 i \kappa Z),
$$

and this is observed in the interferogram of a device such as a Michelson interferometer, Young (two-slit) interferometer, Sagnac, or Mach/Zehnder interferometer. 
It is not possible to describe interferometry in the MaxwellHeaviside theory because the phase factor in that theory is

$$
\exp (i(\boldsymbol{\kappa} \cdot \mathbf{r}-\omega t)) \stackrel{\hat{P}}{\rightarrow} \exp (i(\boldsymbol{\kappa} \cdot \mathbf{r}-\omega t))
$$

Normal reflection is parity inversion, and under the parity inversion operator $\hat{P}$ :

$$
\kappa \rightarrow-\kappa, \quad \mathbf{r} \rightarrow-\mathbf{r},
$$

so the phase (104) does not change under normal reflection in MaxwellHeaviside theory, implying that there is no observable interferogram, contrary to experiment. The effect of parity inversion on the phase (103) however, is as follows:

$$
\begin{aligned}
\hat{P}(\oint \kappa d Z) & =\hat{P}\left(\int_{0}^{Z} \kappa d Z-\int_{Z}^{0} \kappa d Z\right) \\
& =\int_{0}^{-Z} \kappa d Z-\int_{-Z}^{0} \kappa d Z=-\oint \kappa d Z,
\end{aligned}
$$

and the interferogram is predicted and observed experimentally. This is experimental evidence in favor of electrodynamics as a unified field theory in general relativity, in which the $\mathbf{B}^{(3)}$, Evans spin, field is well defined as the following wedge product of tetrads:

$$
B_{\mu \nu}^{(3)}=-i g\left(A^{(1)} \wedge A^{(2)}\right)_{\mu \nu} .
$$

The phase factor in physical optics follows from the Wu-YangDirac phase as the surface integral over the commutator of covariant derivatives [8]:

$$
\Phi=\exp \left(\int\left[D_{\mu}, D_{\nu}\right] d \sigma^{\mu \nu}\right)
$$

Only the $\mathbf{B}^{(3)}$ field

$$
\Phi=\exp \left(\mp \frac{g^{2}}{2} \int\left(A_{Z}^{(1)} A_{Y}^{(2)}-A_{X}^{(2)} A_{Y}^{(1)}\right) d S^{X Y}\right)
$$

contributes to the phase factor in optics and interferometry because the other terms are either zero or oscillatory, averaging to zero over many cycles [8]. The expression of this result in differential geometry is

$$
\oint_{D S} q^{a}=\int_{S} q^{b} \wedge q^{c}=\kappa \int_{S} D \wedge q^{a}
$$




\section{UNIFCATION OF THE FERMAT AND HAMILTON PRINCIPLES IN THE PRINCIPLE OF LEAST CURVATURE, DERIVATION OF WAVE PARTICLE DUALITY AND QUANTUM MECHANICS FROM GENERAL RELATIVITY}

It has been argued that in general relativity the least action in the universe is the Planck constant, a universal constant and the archetypical signature of quantum mechanics. This inference suggests that the well known Fermat principle of least time, which governs optics, and the Hamilton principle of least action, which governs dynamics, can be derived from one principle, to which we refer as the Principle of Least Curvature. The Principle asserts that scalar curvature $R$ is minimized in the equations of motion which govern natural philosophy.

A mathematical expression of the principle is the equation

$$
q_{\mu}^{a}\left(x^{\mu}\right)=e^{i S\left(x^{\mu}\right) / \hbar} q_{\mu}^{a}(0),
$$

which is the equation of motion of the tetrad in general relativity in terms of the spacetime dependent action $S\left(x^{\mu}\right)$. The Evans wave equation [1-3] is obtained from Eq. (111) by applying the operator $\square$ to both sides to give

$$
\square q_{\mu}^{a}\left(x^{\mu}\right)=\square\left(e^{i S\left(x^{\mu}\right) / \hbar} q_{\mu}^{a}(0)\right)=R e^{i S\left(x^{\mu}\right) / \hbar} q_{\mu}^{a}(0) .
$$

Using Eq. (111), we find the Evans lemma and wave equation [1-3],

$$
\square q_{\mu}^{a}\left(x^{\mu}\right)=R q_{\mu}^{a}\left(x^{\mu}\right)=-k T\left(q_{\mu}^{a}\left(x^{\mu}\right)\right)
$$

and identify the scalar curvature as

$$
R=\square\left(e^{i S\left(x^{\mu}\right) / \hbar}\right)
$$

The least possible curvature associated with any particle is

$$
\left|R_{0}\right|=1 / \lambda_{0}^{2}
$$

where $\lambda_{0}$ is its Compton wavelength

$$
\lambda_{0}=\hbar / m c .
$$

The principle of least curvature means that a particle never travels in a precise straight line, because the scalar curvature of a straight line is zero. The least curvature of the particle is defined by the least action $\hbar$ in Eq. (99). This inference means that a particle always has a wave-like 
nature (observed in diffraction and interferometry of matter waves, for example), and so we have derived the de Broglie wave-particle duality from general relativity. The principle of least curvature also means that the phase in optics and dynamics (radiated and matter waves respectively) is an always defined by a Stokes theorem as in Eq. (103) for example, because the phase itself must also have a rotational as well as a translational nature. As we have argued, this inference leads to the first correct explanation of physical optics.

The Evans wave equation is therefore the fundamental equation of quantum mechanics, and is derived from the principle of least curvature in general relativity.

Under local gauge transformation the action is invariant, and so $R$ is invariant. This means that the Aharonov-Bohm effect is a shift from one region of spacetime with a given curvature $R$ to another region of spacetime with the same curvature $R$. This shift is not action at a distance, but could be interpreted as "non-locality" in the sense that one (local) region of spacetime has the same curvature as another region. This is also a possible explanation of entanglement in quantum mechanics. Therefore both entanglement and non-locality are reconciled with the local nature of general relativity. No such reconciliation is possible in special relativity, where there is no concept of $R$, and where there is no principle of least curvature. This means that general relativity is a general theory of natural philosophy. All theories of physics are theories of general relativity. The Dirac equation, for example, is

$$
\begin{aligned}
q_{\mu R}^{a}\left(x^{\mu}\right) & =\exp (i S / \hbar) q_{\mu L}^{a}(0), \\
& \downarrow \hat{P} \\
q_{\mu L}^{a}\left(x^{\mu}\right) & =\exp \left(i S^{*} / \hbar\right) q_{\mu R}^{a}(0),
\end{aligned}
$$

and the time-dependent Schrödinger equation is obtained from

$$
q_{\mu}^{a}(t, \mathbf{r})=e^{i S / \hbar} q_{\mu}^{a}(0, \mathbf{0})
$$

by the following differentiation:

$$
\partial q_{\mu}^{a} / \partial t=(i / \hbar) H q_{\mu}^{a} .
$$

The Hamiltonian is defined [19] by

$$
H=-\partial S / \partial \hbar
$$

Finally, the Heisenberg commutator equation is a cyclic relation between tetrad forms in differential geometry:

$$
q_{\mu \nu}^{c}=\left(q^{a} \wedge q^{b}\right)_{\mu \nu} .
$$


Defining the angular momentum as the tetrad form

$$
J_{\mu}^{a}=J^{(0)} q_{\mu}^{a},
$$

the Heisenberg commutator relation for angular momentum is obtained:

$$
J^{a} \wedge J^{b}=\hbar J^{c},
$$

the starting point for molecular quantum mechanics [19].

Acknowledgments. Craddock Inc., the Ted Annis Foundation, and the Association of Distinguished American Scientists are thanked for funding, and Fellows and Emeriti of AIAS are thanked for several interesting discussions.

\section{REFERENCES}

1. M. W. Evans, Found. Phys. Lett. 16, 367 (2003).

2. M. W. Evans, Found. Phys. Lett. 16, 507 (2003); preprint on www.aias.us.

3. M. W. Evans, Found. Phys. Lett. 17, 25 (2004); 17, 149 (2004); these and other preprints on www.aias.us.

4. L. Felker, ed., The Evans Equations (World Scientific, 2004, in preparation).

5. M. W. Evans, J.-P. Vigier, et al., The Enigmatic Photon (Kluwer Academic, Dordrecht, 1994 to 2002, hardback and softback), in five volumes.

6. M. W. Evans and A. A. Hasanein, The Photomagneton in Quantum Field Theory (World Scientific, Singapore, 1994).

7. M. W. Evans and L. B. Crowell, Classical and Quantum Electrodynamics and the $\mathbf{B}^{(3)}$ Field (World Scientific, Singapore, 2001).

8. M. W. Evans, ed., Modern Nonlinear Optics, a special topical issue in three parts of I. Prigogine and S. A. Rice, series eds., Advances in Chemical Physics (Wiley Interscience, New York, 2001, second edn. and e-book edn.), Vol. 119(1) to 119(3).

9. S. M. Carroll, Lecture Notes in General Relativity, arXiv:grqe/971200019 vl 3 Dec 1997.

10. R. M. Wald, General Relativity (University of Chicago Press, 1994).

11. J. B. Marion and S. T. Thornton, Classical Dynamics of Particles and Systems (HBJ, New York, 1988).

12. L. D. Landau and E. M. Lifshitz, The Classical Theory of Fields (Pergamon, New York, 1975).

13. A. O. Barut Electrodynamics and Classical Theory of Fields and Particles (Macmillan, New York, 1964). 
14. L. H. Ryder, Quantum Field Theory, 2nd edn. (Cambridge University Press, 1996).

15. A. Einstein, The Meaning of Relativity (Princeton University Press, 1921).

16. G. Stephenson, Mathematical Methods for Science Students (Longmans \& Green, London, 1968).

17. T. W. Barrett and D. M. Grimes, eds., Advanced Electromagnetism (World Scientific, Singapore, 1996), pp. $297 \mathrm{ff}$.

18. T. W. Barrett in A. Lakhtakia, ed., Essays on the Formal Aspects of Electromagnetic Theory (World Scientific, Singapore, 1993), pp. 6 ff.

19. P. W. Atkins, Molecular Quantum Mechanics, 2nd edn. (Oxford University Press, 1983).

20. L. O’Raigheartaigh, Rep. Prog. Phys. 42, 159 (1979). 